\section{Physics in Nuclear Medicine}

\author{
S.R. Cherry, J.A. Sorenson, and M.E. Phelps
}

Philadelphia, PA: Elsevier Saunders, 2012, 523 pages, $\$ 115$

Cherry, Sorenson, and Phelps' Physics in Nuclear Medicine is perhaps the best-known textbook on the subject and continues to be the text of choice for many education programs in nuclear medicine technology and nuclear medicine science and for radiology residents. This latest, 2012, edition is the fourth since the original publication in 1980 and brings up to date this now classic introductory text on physics of nuclear medicine.

The content is essentially the same as in the previous edition, except for a new chapter on hybrid scanners. This addition is warranted, given that PET/CT and SPECT/CT have become mainstream imaging devices and that PET/MR scanners were introduced commercially after the 2003 release of the third edition. Otherwise, the outline and order of the material are unchanged. An introductory chapter on what is nuclear medicine is followed by 5 chapters discussing the pertinent fundamental physics that underlies nuclear medicine (basic atomic and nuclear physics, modes of radioactive decay, radioactivity, radionuclide and radiopharmaceutical production, and interaction of radiation with matter). The next 5 chapters cover basic principles of radiation detectors and the associated electronics, nuclear counting and spectrometry, and performance issues and quality assurance related to radiation detection and measurement. The various devices applied clinically (counting systems, planar $\gamma$-cameras, and SPECT and PET scanners), their associated characteristics (performance, image quality), and the basics of filtered backprojection and iterative tomographic SPECT and PET reconstruction are presented in chapters 12 through 18. Discussions of the newer dedicated cardiac SPECT and breast (single-photon and PET) scanners, which are becoming mainstream imaging devices, have been incorporated. Chapters 20 and 21 introduce common digital image processing methods and tracer kinetic modeling and clinical applications, and the last 2 chapters discuss the radiation safety and protection aspects of nuclear medicine, namely MIRD-based internal dosimetry and health physics-related issues (radioactive material handling and disposal, radiation exposure, shielding, monitoring, and protection regulations). The example problems and solutions on various topics found in the prior edition (e.g., radioactivity, interactions, counting statistics, device performance characteristics, and internal dosimetry) are preserved. The 7 appendices contain the same content as before (unit conversions, properties of the elements, characteristics of various relevant radionuclides, relevant material mass attenuation coefficients, dose estimates for selected radiopharmaceuticals, and brief mathematic discussions of the commonly used Fourier transform and convolution techniques).

COPYRIGHT (C 2013 by the Society of Nuclear Medicine and Molecular Imaging, Inc.
The new chapter, 19, begins with a brief discussion of the motivation for hybrid imaging. A review of the basic physics of CT is then presented as a prelude to discussions of the development and current generation of SPECT/CT and PET/CT imaging systems. Descriptions of animal hybrid scanners are included, the rationale being that preclinical combined molecular/ anatomic scanning has become a valuable tool in the development and evaluation of new radiopharmaceuticals, as well as in the assessment of functional and anatomic response to new therapeutic agents before their use in humans. (Preclinical imaging is also mentioned in the chapters on SPECT and PET scanners.) A section describing CT-based attenuation and scatter corrections follows, and the chapter finishes with a brief discussion of the current state of PET/MR scanner development.

Two significant new features of the fourth edition are the transition to color and access to an online version of the book via ExpertConsult.com that comes with the purchase of a hard copy. All chapters, section headings, tables, graphs, and illustrations, as well as some of the clinical images illustrating the functional nature of nuclear medicine/PET, are now in color. The result is improved layout and readability compared with the all-black-and-white previous edition, in my opinion. Online access provides navigation through the text and figures, animated illustrations of key concepts, and interactive calculators and graphs for several equations in the text. Downloading of the figures for use in presentations is enabled.

With the release of the fourth edition, Physics in Nuclear Medicine continues to be a comprehensive and up-to-date nuclear medicine physics and instrumentation textbook, written in such a way as to be an appropriate introduction for both scientist and nonscientist nuclear medicine practitioners. Additional coverage of radiopharmaceutical therapy ( $\beta$ - and $\alpha$-emitters, dosimetry, treatment planning); radioactive patient release (calculations, instructions, regulations); and PET, hybrid imaging, and therapy facility shielding would be useful extensions to the next edition.

\section{William D. Erwin \\ M.D. Anderson Cancer Center \\ Imaging Physics, Unit 1352 \\ P.O. Box 301439 \\ Houston, TX 77230 \\ E-mail:werwin@mdanderson.org \\ Published online May 15, 2013. \\ DOI: $10.2967 /$ jnumed.113.123125 \\ Combined Scintigraphic and Radiographic Diagnosis of Bone and Joint Diseases}

Yong-Whee Bahk

New York, NY: Springer, 2013, 615 pages, \$339

Bone is no longer considered a static structure. It alters as osteoblasts are mineralized and osteoclasts are resorbed. Combined 